\title{
Computational Structure Prediction Provides a Plausible Mechanism for Electron Transfer by the Outer Membrane Protein Cyc2 from Acidithiobacillus ferrooxidans
}

\author{
Virginia Jiang ${ }^{1}$, Sagar D. Khare² and Scott Banta ${ }^{1, *}$
}

${ }^{1}$ Department of Chemical Engineering, Columbia University in the City of New York, New York, NY 10027, USA

${ }^{2}$ Department of Chemistry and Chemical Biology, Rutgers, The State University of New Jersey, Piscataway, NJ 08854, USA

${ }^{*}$ Corresponding author. Tel: +1-212-854-7531. Fax: +1-212-854-3054. E-mail address: sbanta@columbia.edu,

Total manuscript pages (without figures) - 26

Supplementary material pages- 14 including 14 supplementary figures

Number of tables- 0

Total number of figures- 4

Description of supplementary information - A "Supplementary information" file has been uploaded which contains primary sequence information, topology files, multiple sequence alignments, additional structural figures, and additional score vs RMSD plots. 


\section{Abstract:}

Cyc2 is the key protein in the outer membrane of Acidithiobacillus ferrooxidans that mediates electron transfer between extracellular inorganic iron and the intracellular central metabolism. This cytochrome $\mathrm{c}$ is specific for iron and interacts with periplasmic proteins to complete a reversible electron transport chain. A structure of Cyc2 has not yet been characterized experimentally. Here we describe a structural model of Cyc2, and associated proteins, to highlight a plausible mechanism for the ferrous iron electron transfer chain. A comparative modeling protocol specific for trans membrane beta barrel (TMBB) proteins in acidophilic conditions $(\mathrm{pH} \sim 2)$ was applied to the primary sequence of Cyc2. The proposed structure has three main regimes: extracellular loops exposed to low-pH conditions, a TMBB, and a N-terminal cytochrome-like region within the periplasmic space. The Cyc2 model was further refined by identifying likely iron and heme docking sites. This represents the first computational model of Cyc2 that accounts for the membrane microenvironment and the acidity in the extracellular matrix. This approach can be used to model other TMBBs which can be critical for chemolithotrophic microbial growth.

Keywords: transmembrane beta barrel, heme protein, iron oxidation, Rosetta, chemolithoautotrophy

\section{Importance of work (50-75 words):}

Acidithiobacillus ferrooxidans can oxidize both iron and reduced sulfur compounds and plays a key role in metal sulfide ore bioleaching used for the industrial recovery of metals. 
A. ferrooxidans has also been explored as a potential organism for emerging technologies such as e-waste recycling and biofuel production. Synthetic biology efforts are hampered by lack of knowledge about the mechanisms of iron oxidation and reduction, which is mediated by the Cyc2 transmembrane beta barrel (TMBB) protein.

\section{Abbreviations:}

REU - Rosetta Energy Unit

TMBB - trans-membrane beta barrel protein

Cyc2 - gene name for outer membrane cytochrome c protein of $A$. ferrooxidans

Cyc1 - gene name for cytochrome c552 protein of $A$. ferrooxidans

Rcy - rusticyanin protein of $A$. ferrooxidans

RMSD - root mean square deviation of atomic positions, given in angstroms $(\AA)$

OPM - Orientations of Proteins in Membranes database (58)

MIB - Metal lon-Binding Site Prediction and Docking Server (53) 


\section{Introduction}

Acidithiobacillus ferrooxidans is a gram-negative acidophilic chemolithoautotroph that exhibits the highest growth rates in the range between $\mathrm{pH} 1.8$ and $2.2(37)$. It is an important electrochemically active bacterium (EAB) involved in mineral bioleaching due to its ability to oxidize both ferrous iron and reduced sulfur compounds. This capability has been leveraged in industrial processes where the resulting oxidized iron is exploited for the recovery of copper and other valuable metals from sulfidic ores $(67 ; 85)$ (Fig. 1a). In addition to its role in industrial "biomining" there has been increasing interest in developing $A$. ferrooxidans for other applications such as electronic waste recycling (72), and as a unique chassis organism for biofuel production. Genetically modified $A$. ferrooxidans cells have been engineered to create biochemicals from $\mathrm{CO}_{2}$ using electrochemically reduced iron as the sole energy source, and these types of "electrofuels" platform provide a promising route towards enabling the conversion of renewable electrical energy into the chemical energy of transportation fuels $(51 ; 42 ; 10)$.

When not growing on sulfur, $A$. ferrooxidans cells are able to obtain all of their metabolic energy via the extracellular oxidation of ferrous iron. Thus iron supports mediated electron transfer (MET), enabling the cells to grow planktonically and the iron can be reduced by solid surfaces or by electrodes. The cells can also demonstrate direct electron transfer (DET) where they are able to grown on solid surfaces including electrodes, and the electrons can flow directly into the cellular metabolism (18). Once inside the cells, the electrons flow through two pathways. In the thermodynamically uphill pathway, electrons are used to regenerate the reducing equivalents (NADP(H)). In the thermodynamically downhill pathway, oxygen is the final electron acceptor and protons are used to produce water, enabling the cells to produce ATP and to maintain 
an internally neutral $\mathrm{pH}(77)$. When growing on reduced sulfur compounds, oxygen usually serves as the terminal electron acceptor. However, under anaerobic conditions, ferric iron can also serve as an electron acceptor $(73 ; 48)$. Therefore, under these conditions, the flow of electrons is reversed and the cells become iron reducers. Thus, unlike many other $\mathrm{EAB}$, the electron transfer machinery in $A$. ferrooxidans is reversible.

The proteins involved in electron transfer into and out of the cells are encoded in the rus operon; which includes an outer membrane cytochrome c protein (Cyc2), a periplasmic blue copper protein (rusticyanin, or Rcy), a periplasmic cytochrome c4 (Cyc1), and an inner membrane aa3-type cytochrome c oxidases (7; 99) (Fig. 1b). The periplasmic proteins Rcy and Cyc1 have previously been crystallized and characterized $(87 ; 1)$, and the surface interactions at the interface between Rcy and Cyc1 have been determined (66). The Cyc2 protein (485 amino acids, MW 52.4 kDa, Fig. S1, Fig. S2) is the critical outer transmembrane protein that serves as the extracellular interface for electron transfer necessary for iron oxidation or reduction $(97 ; 18)$. Overexpression of the cyc2 gene increases Fe(II) oxidation (54), as does overexpression of the rus gene that encodes Rcy (36). 3-D structural information for Cyc2 has not yet been reported, likely due to experimental difficulties, and this hampered our mechanistic understanding of this key energetic pathway.

Computational modeling of the Cyc2 protein structure has been difficult as there are few homologous membrane protein structures available based on sequence similarity that have been crystalized. The development of robust computational methodologies that can account for the membrane microenvironment is a growing field of research $(2 ; 69)$. Outer membrane proteins in gram-negative bacteria typically 
contain transmembrane beta barrel (TMBB) domains that act as pores to control intercellular transport (15). As Cyc2 is located in the outer membrane, it is likely a TMBB. To date, most transmembrane protein modeling efforts have focused on alphahelical transmembrane proteins, which are most often found in the inner cell membrane (44; 12; 13; 89); however modeling and designing TMBBs is an emerging area of research. The 3D structural prediction of TMBBs from primary amino acid sequence is hindered by limited structural information, however several tools have been developed for both comparative (CM) and de novo modeling of TMBBs $(75 ; 30 ; 3 ; 32 ; 27 ; 81)$. Computational tools for de novo design of TMBBs have also been developed $(79 ; 86)$. Additionally, as $A$. ferrooxidans require highly acidic environments, modeling methods applied to Cyc2 should account for pH-dependent protein conformations.

Experimental evidence indicates that Cyc2 has several cofactors and binding interactions. Ferrous iron does not accumulate significantly within the cells, and the ferrous oxidation site is localized in the outer membrane facing the exterior of the cell (38). Cyc2 domains have been experimentally demonstrated to project from the cells into the extracellular matrix (97). The $\mathrm{N}$-terminus of the protein primary sequence contains one $c$-type cytochrome motif $\mathrm{CXXCH}$, where a heme likely is covalently bonded to the protein by two thioether bonds with the cysteine sulfur atoms and an $\mathrm{H}$ bond to the nitrogen on the histidine ring (4). Purified Cyc2 shows a monohemic signature when characterized by spectrophotometry (97).

To create structural models of Cyc2 and related outer membrane proteins, there is a need to develop TMBB-specific modeling tools so that new insights can be gained into how electrons are transferred from external sources to interior cellular metabolisms. 
Rosetta, a widely used biomolecular modeling and design package, contains computational frameworks for comparative modeling (RosettaCM)(13), membrane protein design (RosettaMP)(2), and pKa calculations (Rosetta-pH)(43). Additionally, there are existing Rosetta protocols for determining protein-protein docking interactions (Rosetta Dock). RosettaCM has a well-documented protocol for comparative modeling of alpha helical transmembrane proteins but no specialized protocol for TMBB structures currently exists (25).

In this manuscript, these existing Rosetta function platforms were combined to develop a TMBB model for the Cyc2 cytochrome c protein of $A$. ferrooxidans. The modeling framework was adjusted for the environmental conditions specific for $A$. ferrooxidans. Putative metal binding sites in the structure were identified, and docking of the Rus and Cyc1 proteins was explored. This new structural ensemble can be used to provide insights into the formation of the natural "molecular wire" that enables reversible electron exchange with inorganic materials in the environment.

\section{Results}

The Rosetta membrane protein comparative modeling protocol has been primarily used to model alpha helical transmembrane proteins (96). The protocol involves a low-resolution step where amino acid sidechains are represented by a single pseudo-atom placed at the geometric centroid of all atom positions (centroid mode) followed by a high-resolution step in which all atoms including hydrogen atoms are explicitly modeled (full-atom mode). Interactions in the low- and high-resolution steps are described using the centroid and full atom energy functions, respectively. To expand the scope of this methodology to include TMBB proteins like Cyc2, we altered the low- 
resolution centroid score function by removing alpha helical membrane protein-specific terms from the low-resolution centroid score function (mp_nonhelix, mp_termini, mp_tmproj) (Fig. S3). Next, the full atom score function was modified to franklin2019 weights, which includes fa_water_to_bilayer, a score term that accounts for membraneembedded protein transfer free energy (2). We tested the impact of these changes on TMBB structure prediction on three test proteins as described in the Methods/SI and found significant improvements in prediction performance (Fig. S4, S5).

With a protocol for modeling TMBB proteins in hand, we turned to the Cyc2 primary protein sequence from Acidithiobacillus ferrooxidans ATCC 23270 (Fig. S1). The modified RosettaCM protocol for TMBB proteins was used to model Cyc2 from PDB IDs 2ov4 and 4rjw (65; 80), homologous 16-stranded TMBB proteins in the OPM database (Fig. 2a). As the outer membrane of $A$. ferrooxidans faces an extracellular matrix at $\mathrm{pH} 2$, the full atom score function was modified to include terms fa_elec and e_pH and the Rosetta-pH package was initialized to match known $\mathrm{pH}$ values for the extracellular matrix. Salt bridges between side chains that were present at $\mathrm{pH} 7$ were not predicted at $\mathrm{pH} 2$ (Fig. S6), which is consistent with experimentally observed salt bridge formation behavior (64). The lowest-scoring model was scored by MolProbity where it had an overall MolProbity score of 2.01 , falling into the $73^{\text {rd }}$ percentile of all available structures based on assessments of protein geometry. Ramachandran favored rotamers comprised $98.62 \%$ of the residues, while Ramachandran poor rotamers comprised $0.27 \%$ of the protein. The Cyc2 model had a ProQ3 S-score of 0.36, which is within the predicted range of scores for proteins in the CASP11 test set 
$(0.40 \pm 0.12)(83 ; 82)$. The sequence for Cyc2 was accessed in PSIPRED for secondary structure and domain topology (Fig. S7) $(57 ; 17)$.

The resulting model is a monomeric 16-stranded TMBB that spans the membrane, with a cytochrome-like domain consisting of several short alpha helices and a bound heme group that is fused to the beta barrel at the $\mathrm{N}$-terminus of the protein (Fig. 2b). The TMBB topology is similar to other 16-stranded TMBB porins. The model has an annular pore at the apical side facing the periplasmic space, with flexible loops facing the acidic extracellular environment and the $\mathrm{N}$-terminus cytochrome-like domain protruding into the periplasm. The majority of the protein contains beta-sheet rich domains, as expected for an outer membrane TMBB protein.

Potential metal binding sites were then explored in the Cyc2 model structure. Homologous binding locations were identified against all known metal binding structures in the RCSB PDB using the Metal lon-Binding Site Prediction and Docking Server (MIB) (Fig. S8). The binding sites with evolutionarily conserved residues as measured by ClustalOmega in ten related iron-oxidizing bacteria were identified and used as initial binding sites for metal ion docking (60) and metal chelation geometry was optimized using Rosetta (Fig. S9). Two binding sites were identified. The most likely binding location was at residues $\mathrm{H} 119, \mathrm{D} 137, \mathrm{D} 138$, and was homologous to a ribonucleotide reductase protein from Corynebacterium ammoniagenes (PDB ID: 1kgo)(34). Calculated binding geometries are square planar, with angle RMSDs of $6.2^{\circ}$ (Fig. 2c). An additional probable second binding site was identified at Y262 and D308 and was homologous to a site in YmdB, a phosphodiesterase from Bacillus subtilis (PDB ID: 
4b2o)(22). The binding geometry of this site is tetrahedral, with angle RMSDs of $7.3^{\circ}$ (Fig. 2d).

To further investigate electron transfer pathways in the predicted Cyc2 structure, the model was further refined by docking the heme $\mathrm{C}$ binding domain via homology to a heme binding protein involved in the electron transfer pathway in Vibrio

parahaemolyticus by aligning the $\mathrm{CXXCH}$ motifs (PDB ID: 2zzs)(14). Calculated binding geometries are octahedral, with angle RMSDs of $2.7^{\circ}$ from ideal (Fig. 2e).

The predicted ligand and ion binding interactions with the model Cyc2 structure are consistent with prior experimental work in $A$. ferrooxidans. The structure has two internally bound iron atoms in the barrel region and one bound heme $\mathrm{c}$ in the cytochrome-like region (Fig. 2b), which corresponds with ligands that have been coeluted with Cyc2 during purification (6). Heme-protein interactions fall within experimentally measured parameters. The imidazole ring of $\mathrm{H} 16$ is $2.1 \mathrm{~A}$ from the docked central iron in the heme C (Fig. 2e), which corresponds to the experimentally determined interaction radius $(68 ; 101)$. Metal residue chelating geometries around the bound irons have low angle RMSD values below $10^{\circ}$, indicating that structural geometries of the coordination spheres approach the ideal metal ion environments within the protein (95).

Next, docking was performed to find possible interfaces between Cyc2 and its downstream partners in the electron transfer chain, Rcy and Cyc1. These are periplasmic proteins with crystallographic structures available (PDB ID: 1rcy and 1h10) $(87 ; 1)$. A structural model of the Rcy-Cyc1 complex has been previously predicted: Abergel et. al determined a probable interface between of rusticyanin and Cyc1 (Fig. 
S10) We confirmed this interface was plausible by using ClusPro for FFT patch-based docking and refining using local Rosetta docking. The modeled Rcy-Cyc1 complex was then docked with Cyc2 to produce an interface score vs RMSD plot with a narrow energy funnel. Patches were identified by regions of conservation with electron transferactive or chelating residues on Rcy. D58 was identified as a probable patch location on Rcy, with $\mathrm{H} 57$ as a possible redox center. A similar hydrogen bonding interface was identified on the docked model of Cyc2 to Rcy (Fig. S10).

The protein-protein docking results are consistent with experimental findings. Docking simulations with Rosetta confirmed previous modeling efforts to dock Rcy and Cyc1. H57 and D58 in Rcy had previously been thought to be a possible copper binding site prior to crystallographic analysis (78), as the residues on Rcy in those positions align with known copper binding sites on azurin from Pseudomonas aeruginosa. By contrasting extended X-ray absorption fine structure (EXAFS) on oxidized and reduced forms of Rcy, it has been shown that there are multiple rigid electron transfer sites potentially contributing to the higher reduction potential of Rcy (35). The authors identified electron transfer interactions involving nitrogen atoms on histidine imidazole rings ( $\mathrm{H} 57, \mathrm{H} 85$, and $\mathrm{H} 143$ ), which had all previously been thought to be involved in copper coordination (28). However, the X-ray crystal structure of Rcy showed that two of the identified histidines coordinate copper binding (H85 and H143). Large interatomic distances between $\mathrm{H} 57$ and the copper ion make it unlikely that all the histidines chelate the same copper ion. We hypothesize that $\mathrm{H} 57$ could instead play a role in the electron transfer pathway between Cyc2 and Rcy, and D58 may be involved in stabilizing the 
interface between Cyc2 and Rcy due to its hydrogen bonding ability (66). Therefore, this residue patch is thought to be most likely Cyc2-Rcy interface on Rcy.

The docked structures were used to identify putative electron transfer pathways through the full Cyc2-Rcy-Cyc1 complex (Fig. 3a). Emap, a platform that identifies potential electron tunneling pathways via using graph theory to map node distances between aromatic amino acids, was used to discover potential transfer pathways (80). This analysis indicates there exists a probable electron hopping pathway within the full model complex from the Cyc2 model structure to the experimentally verified Rcy-Cyc1 complex (Fig. 3b). The residues implicated in electron transfer with this model of Cyc2 are primarily phenylalanines and tyrosines, a phenomenon which has been experimentally observed in yeast cytochrome $\mathrm{c}$ via point mutations to residues without electron-transfer active moieties (52). The computational predictions for Cyc2 account for known electron transfer interactions, such as electron hopping between Rcy H143 and the Rcy docked copper ion. Additionally, there are experimentally predicted electron interactions in Cyc1 between Y143, Y42, and the most downstream bound heme (heme 1185) (1). The shielding provided by the tyrosine residues around Cyc1 heme 1185 indicates that heme 1185 is less solvent-accessible than heme 1184 and therefore heme 1185 is the higher potential driving force in the Cyc1 cytochrome.

Possible Fe(II) transient binding sites were identified using regions of negative potential as calculated by Poisson-Boltzmann scores (Fig. 4a, 4b). Within this model, a putative iron binding site on the exterior of the cell may be present, with a topological pocket in a region of high negative potential. However, more experimental evidence will be needed to verify that this is the site of iron binding in Cyc2. When viewed from the 
periplasmic side of the protein, the solvent-exposed pore extending through the protein has highly negative Poisson-Boltzmann potential (Fig. 4c). This is consistent with porins selective for cations; porins selective for anions have inner pores with positive PoissonBoltzmann potentials, while porins with no ion selectivity show no clear pattern in Poisson-Boltzmann potential (Fig. S11). Thus the negative potential of the pore suggests Cyc2 may also facilitate cation transport.

\section{Discussion}

Cyc2 is the key outer membrane protein in A. ferrooxidans that enables electron transfer from extracellular inorganic materials $(19 ; 49 ; 47)$. To create a structural model, a modified score function was developed which allows Rosetta comparative modeling protocols to be extended to TMBBs. This procedure was used to produce a new structural model of the Cyc2 protein structure. The heme ligand was docked onto the protein and putative metal binding sites were identified. The modelled protein was docked onto Rcy-Cyc1, leading to a model of the electron transfer pathway in $A$. ferrooxidans that is consistent with known experimental details.

EAB all share the ability to exchange electrons across their insulating outer membrane(s), although different mechanisms have evolved depending on the specific metabolism of the species. In every case, electron transfer is mediated by cofactorcontaining outer membrane proteins $(91 ; 93)$. For example, Shewanella oneidensis is a metal oxide-reducing gram-negative bacterium, which uses a combination of methods for electron uptake but is dominated by flavin-dependent MET $(45 ; 70 ; 94)$. The structure of the main S. oneidensis transmembrane electron conduit MtrCAB is trimeric: 
one peptide protrudes into the extracellular matrix, one serves as a transmembrane porin, and one is a multi-heme cytochrome (26). There is a directional hierarchy in the electron transfer pathway due to the presence of multiple chained hemes (29).

Another species of EAB, Geobacter sulfurreducens, transfers electrons from mineral surfaces using conductive pili $(76 ; 61)$. Over 110 genes coding for putative ctype cytochromes have been identified in G. sulfurreducens, resulting in many parallel electron transfer pathways (63). Two major trimeric conduits have been identified, OmaB-OmbB-OmcB and OmaC-OmbC-OmcC, both of which consist of two cytochromes and one transmembrane porin $(56 ; 55)$. G. sulfurreducens electron transfer can be bidirectional, but experimental findings suggest the cathodic electron transfer, where electrons move from interior metabolism to the exterior matrix, may take place via an alternative non-heme protein (33).

G. sulfurreducens and $S$. oneidensis have the most extensively studied porincytochrome systems which are both trimeric systems. There are also some dimeric porin-cytochrome systems, including Sideroxydans lithotrophicus, and Rhodopseudomonas palustris. A. ferrooxidans lacks a multimeric porin-cytochrome complex; rather, Cyc2 is a simpler monomeric fused porin-cytochrome structure that enables electrons to flow in or out of the cell depending on the orientation of the driving force (91).

This A. ferrooxidans Cyc2 structure may be useful for developing structures for homologous TMBB electron transfer complexes. For instance Cyt572, a functionally and structurally similar protein with low sequence identity to Cyc2, has been identified in 
other acidophilic iron-oxidizing bacterial communities comprised of Leptospirillum spp (39). And the cyc2 gene is conserved across a range of neutrophilic iron-oxidizing bacterial mats $(11 ; 62 ; 41)$. Currently, there are no crystallized structures of functionally homologous fused porin-cytochromes found in the outer membrane in the OPM databank (58).

It has been suggested that the electron transfer from Fe(II) to Cyc2 occurs via a transient encounter-Michaelis complex $(16 ; 50)$, such that there is a transient ironbinding site on the outside face of Cyc2 that serves as the initial binding site for $\mathrm{Fe}(\mathrm{II})$ oxidation and electron transfer. Such transient interactions are not well-captured by crystallography, so there are no homologous binding geometries in the Protein Data Bank. As shown in Fig 4, there is a putative metal binding site on the Cyc2 protein structure that is close to the outer membrane surface. An additional poorly understood feature of Cyc2 is the binding site specificity between different metal ions. In addition to growth on $\mathrm{Fe}(\mathrm{II})$ it has also been shown that the cells can oxidize uranium from uranous to uranyl, suggesting that the Cyc2 electron transport chain can carry electrons from both uranium and iron $(23 ; 90)$. It has also been demonstrated that the cells cannot grow on vanadium (III). However when a small amount of iron is included, growth on $\mathrm{V}(\mathrm{III})$ can occur where iron is used as a redox mediator between vanadium and the cells (51). The mechanism for this metal selectivity is not yet known and further experimental work will be necessary to validate the location of the metal binding pocket.

\section{Conclusion}


A comparative modeling protocol was applied to the Cyc2 sequence to obtain a modular model with a cytochrome-like heme c binding domain, a flexible linker, and a membrane-embedded beta barrel. Iron-binding sites within Cyc2 were identified by homology. Protein-protein docking was performed to determine likely interfaces between Cyc2, Rcy, and Cyc1 to produce an "conducting wire" spanning from the extracellular matrix through the outer membrane into the periplasm. Electron hopping distances in the pathway were calculated using graph theory, and it was determined that there is a plausible electron transfer pathway from $\mathrm{Fe}(\mathrm{II})$ through the model of Cyc2 and to the docked Rcy-Cyc1 complex. This model is the only known computational model of Cyc2 that accounts for the membrane microenvironment and the acidity levels in the extracellular matrix and aligns with experimental findings. This model of Cyc2 provides a blueprint for the genetic engineering of Cyc2. And the modeling approach may be useful for modeling other TMBBs which can be critical for chemolithotrophic microbial growth.

\section{Materials and Methods}

Membrane embedded alpha helix specific terms were removed from the 2006 Yarov-Yarovoy centroid score function for membrane proteins(96). The modified set of weight terms were used in the stages prior to full atom refinement of a comparative modeling protocol (Fig. S3). The full atom score function used for comparative modeling was franklin2019, part of the RosettaMP framework that has been validated for changes 
in Gibbs free energy given a mutation (DDGmut) and native structure identification of TMBBs (2).

The franklin2019 full atom core function was used for all trajectories. To account for the acidic environment on the exterior of the cell, the flags "-pH_mode" and "value_pH 2" from Rosetta-pH were used to calculate the pKa values assuming media of $\mathrm{pH} 2$ and set protonation states of residues outside the membrane (43). The score terms fa_elec and $e \_p H$ was set to 1.0 and added to the franklin2019 score function. Spanfiles to determine the transmembrane regimes were from Boctopus, a server that uses support vector machines (SVMs) and Hidden Markov Models (HMMs) to calculate TMBB topologies (31). Constraints based on evolutionary similarity were generated from GREMLIN (Fig. S12). Threaded models were based on GREMLIN calculations of evolutionary similarity (71). These weights files were then used in the 2018 RosettaCM protocol (13).

The same protocol was used for comparative modeling of Cyc2 (UniPROT: B7JAQ7_ACIF2) from Acidithiobacillus ferrooxidans (strain ATCC 23270 / DSM 14882 / CIP 104768 / NCIMB 8455). Signal peptides in the sequence were detected by SignalP 5.0, a neural network signal peptide detection server (5). Threaded models were generated from 16-stranded TMBBs with known structures identified using GREMLIN as having evolutionarily conserved and coevolved regions with above $95 \%$ probability of residue-residue contacts $(9 ; 40 ; 71)$ (Fig. S12). Outer membrane porin proteins OprP and OprO from the gram-negative bacterium Pseudomonas aeruginosa (respective PDB codes $20 v 4$ and 4rjw) were used as homologs $(65 ; 80)$. OprP and OprO are trimeric porins, but earlier molecular weight characterizations of Cyc2 indicated that it is 
a monomeric protein(6), so one subunit of each of the Pseudomonas aeruginosa OMBB proteins was used. These threaded models were hybridized, and the new weight sets were used to $a b$ initio fold regions with low structural or sequence homology in a membrane environment. The model used DPLC (1,2-dilauroyl-sn-glycero-3phosphocholine), a common component of gram-negative bacteria outer membranes with membrane depth of $30 \mathrm{~A}$. The resulting models were then minimized using MPRelax with the franklin2019 score function. 100 trajectories were run of the CM protocol and the three lowest-scoring models were checked using MolProbity (92). The model with the most favorable protein geometry was selected as the most likely.

The Cyc2 sequence has a known heme c binding domain (74) that was modeled based on homology from a cytochrome c identified by GREMLIN to have the highest coverage and most probable residue-residue interaction (PDB code 2zzs) (14). The cytochrome c554 was from Vibrio parahaemolyticus, a bacterium that obtains energy for growth from the oxidation of ammonia to nitrite (14). Heme c was docked via homology and verified by measuring the heme iron-imidazole distances. Both $\mathrm{Fe}(\mathrm{II})$ and $\mathrm{Fe}(\mathrm{III})$ docking sites were found by homologous templates in the PDB using the Metal lonBinding Site Prediction and Docking Server (MIB) (53). Potential metal binding sites were searched in BLAST against proteins with similar functions in other organisms. The ten most similar protein sequences were compared for conserved residues in the potential iron binding sites, and any sites without similar residues conserved across species of iron oxidizing bacteria were discarded (Fig. S8, S9). Amino acid side chains in metal binding sites in conserved regions were repacked in PyRosetta using the flag "in:auto_setup_metals" and the metalbinding_constraint weight set to 1.0 to arrive at 
more favorable metal chelating geometries as calculated by metalbinding_constraint score term (88) and relaxed using 100 trajectories (Fig. S13). These metal geometries were verified with the CheckMyMetals webserver (100).

Abergel created docked models of the periplasmic proteins in the electron transfer chain downstream from Cyc2. Rcy (PDB code 1rcy) is an oxidizing cupredoxin that likely serves as the immediate downstream partner in the electron transport chain from Cyc2 (87). Cyc1 (PDB code 1h10) is downstream of Rcy and serves as a "tuner" for mediating electron transfer (1). From experimentally determined interactions, fast Fourier transform (FFT) docking was performed in ClusPro between Rcy and Cyc1 (46; 84) and refined using ROSIE Docking (59) (Fig. S14). Interactions between Rcy and Cyc2 were determined from conserved regions on the Rcy faces known not to interact with Cyc1. Cyc2 was docked onto the likely Rcy-Cyc1 complex (1) using ClusPro for global docking. All residues in the transmembrane domains of Cyc2 were assigned a repulsive score to favor Rcy docking with the cytochrome region. The best-scoring model was processed in ROSIE Docking2 for refinement. Electron transfer across the three docked proteins was calculated using eMap, a server that predicts electron tunneling through electron transfer active (ETA) moieties using graph theory (80).

To model the transient initial binding of $\mathrm{Fe}(\mathrm{II})$ on the outermost loops of Cyc2, electron potential was calculated using a Poisson-Boltzmann model and the regions with the most negative potential were identified( $8 ; 24)$. 


\section{Acknowledgements}

The authors gratefully acknowledge financial support from Rosetta Commons and NSF

REU Award 1950697 as well as the U.S. Army Research Office (grant W911NF-18-1-

0239).

\section{References}

1. Abergel C, Nitschke W, Malarte G, Bruschi M, Claverie J-M, Giudici-Orticoni M-T (2003) The Structure of Acidithiobacillus ferrooxidans c4-Cytochrome. Structure 11:547-555.

2. Alford RF, Fleming PJ, Fleming KG, Gray JJ (2020) Protein Structure Prediction and Design in a Biologically Realistic Implicit Membrane. Biophys J 118:2042-2055.

3. Alford RF, Koehler Leman J, Weitzner BD, Duran AM, Tilley DC, Elazar A, Gray JJ (2015) An Integrated Framework Advancing Membrane Protein Modeling and Design. PLoS Comput Biol 11:e1004398.

4. Allen JW, Leach N, Ferguson SJ (2005) The histidine of the c-type cytochrome $\mathrm{CXXCH}$ haem-binding motif is essential for haem attachment by the Escherichia coli cytochrome c maturation (Ccm) apparatus. Biochem J 389:587-592.

5. Almagro Armenteros JJ, Tsirigos KD, Sonderby CK, Petersen TN, Winther O, Brunak S, von Heijne G, Nielsen H (2019) SignalP 5.0 improves signal peptide predictions using deep neural networks. Nat Biotechnol 37:420-423.

6. Appia-Ayme C, Bengrine A, Cavazza C, Giudici-Orticoni MT, Bruschi M, Chippaux M, Bonnefoy V (1998) Characterization and expression of the co-transcribed cyc1 and cyc2 genes encoding the cytochrome c4 (c552) and a high-molecular-mass cytochrome c from Thiobacillus ferrooxidans ATCC 33020. FEMS Microbiol Lett 167:171-177.

7. Appia-Ayme C, Guiliani N, Ratouchniak J, Bonnefoy V (1999) Characterization of an operon encoding two c-type cytochromes, an aa(3)-type cytochrome oxidase, and rusticyanin in Thiobacillus ferrooxidans ATCC 33020. Appl Environ Microbiol 65:4781-4787.

8. Baker NA, Sept D, Joseph S, Holst MJ, McCammon JA (2001) Electrostatics of nanosystems: application to microtubules and the ribosome. Proc Natl Acad Sci U S A 98:10037-10041.

9. Balakrishnan S, Kamisetty H, Carbonell JG, Lee S-I, Langmead CJ (2011) Learning generative models for protein fold families. Proteins: Structure, Function, and Bioinformatics 79:1061-1078.

10. Banerjee I, Burrell B, Reed C, West AC, Banta S (2017) Metals and minerals as a biotechnology feedstock: engineering biomining microbiology for bioenergy applications. Curr Opin Biotechnol 45:144-155.

11. Barco RA, Emerson D, Sylvan JB, Orcutt BN, Jacobson Meyers ME, Ramirez GA, Zhong JD, Edwards KJ (2015) New Insight into Microbial Iron Oxidation as 
Revealed by the Proteomic Profile of an Obligate Iron-Oxidizing Chemolithoautotroph. Appl Environ Microbiol 81:5927-5937.

12. Barth P, Senes A (2016) Toward high-resolution computational design of the structure and function of helical membrane proteins. Nat Struct Mol Biol 23:475480.

13. Bender BJ, Cisneros A, 3rd, Duran AM, Finn JA, Fu D, Lokits AD, Mueller BK, Sangha AK, Sauer MF, Sevy AM, Sliwoski G, Sheehan JH, DiMaio F, Meiler J, Moretti R (2016) Protocols for Molecular Modeling with Rosetta3 and RosettaScripts. Biochemistry 55:4748-4763.

14. Bergmann DJ, Hooper AB, Klotz MG (2005) Structure and Sequence Conservation of hao Cluster Genes of Autotrophic Ammonia-Oxidizing Bacteria: Evidence for Their Evolutionary History. Applied and Environmental Microbiology 71:53715382.

15. Bishop RE (2008) Structural biology of membrane-intrinsic beta-barrel enzymes: sentinels of the bacterial outer membrane. Biochim Biophys Acta 1778:18811896.

16. Blake RC, 2nd, Shute EA (1994) Respiratory enzymes of Thiobacillus ferrooxidans. Kinetic properties of an acid-stable iron:rusticyanin oxidoreductase. Biochemistry 33:9220-9228.

17. Buchan DWA, Jones DT (2019) The PSIPRED Protein Analysis Workbench: 20 years on. Nucleic Acids Res 47:W402-W407.

18. Castelle C, Guiral M, Malarte G, Ledgham F, Leroy G, Brugna M, Giudici-Orticoni MT (2008) A new iron-oxidizing/O2-reducing supercomplex spanning both inner and outer membranes, isolated from the extreme acidophile Acidithiobacillus ferrooxidans. J Biol Chem 283:25803-25811.

19. Corbett CM, Ingledew WJ (1987) Is Fe3+/2+cycling an intermediate in sulphur oxidation by Fe2+-grownThiobacillus ferrooxidans. FEMS Microbiology Letters 41:1-6.

20. Delattre AS, Clantin B, Saint N, Locht C, Villeret V, Jacob-Dubuisson F (2010) Functional importance of a conserved sequence motif in $\mathrm{FhaC}$, a prototypic member of the TpsB/Omp85 superfamily. FEBS J 277:4755-4765.

21. Dhakshnamoorthy B, Raychaudhury S, Blachowicz L, Roux B (2010) Cationselective pathway of OmpF porin revealed by anomalous X-ray diffraction. J Mol Biol 396:293-300.

22. Diethmaier C, Newman JA, Kovacs AT, Kaever V, Herzberg C, Rodrigues C, Boonstra M, Kuipers OP, Lewis RJ, Stulke J (2014) The YmdB phosphodiesterase is a global regulator of late adaptive responses in Bacillus subtilis. J Bacteriol 196:265-275.

23. DiSpirito AA, Tuovinen $\mathrm{OH}$ (1982) Uranous ion oxidation and carbon dioxide fixation by Thiobacillus ferrooxidans. Archives of Microbiology 133:28-32.

24. Dolinsky TJ, Nielsen JE, McCammon JA, Baker NA (2004) PDB2PQR: an automated pipeline for the setup of Poisson-Boltzmann electrostatics calculations. Nucleic Acids Res 32:W665-667.

25. Duran AM, Meiler J (2018) Computational design of membrane proteins using RosettaMembrane. Protein Sci 27:341-355. 
26. Edwards MJ, White GF, Butt JN, Richardson DJ, Clarke TA (2020) The Crystal Structure of a Biological Insulated Transmembrane Molecular Wire. Cell 181:665673 e610.

27. Franklin MW, Nepomnyachyi S, Feehan R, Ben-Tal N, Kolodny R, Slusky JS (2018) Evolutionary pathways of repeat protein topology in bacterial outer membrane proteins. Elife 7.

28. Hall JF, Hasnain SS, Ingledew WJ (1996) The structural gene for rusticyanin from Thiobacillus ferrooxidans: cloning and sequencing of the rusticyanin gene. FEMS Microbiol Lett 137:85-89.

29. Harada E, Kumagai J, Ozawa K, Imabayashi S, Tsapin AS, Nealson KH, Meyer TE, Cusanovich MA, Akutsu H (2002) A directional electron transfer regulator based on heme-chain architecture in the small tetraheme cytochrome $c$ from Shewanella oneidensis. FEBS Letters 532:333-337.

30. Hayat S, Elofsson A (2012) Ranking models of transmembrane beta-barrel proteins using Z-coordinate predictions. Bioinformatics 28:i90-96.

31. Hayat S, Peters C, Shu N, Tsirigos KD, Elofsson A (2016) Inclusion of dyad-repeat pattern improves topology prediction of transmembrane beta-barrel proteins. Bioinformatics 32:1571-1573.

32. Hayat S, Sander C, Marks DS, Elofsson A (2015) All-atom 3D structure prediction of transmembrane beta-barrel proteins from sequences. Proc Natl Acad Sci U S A 112:5413-5418.

33. Heidary N, Kornienko N, Kalathil S, Fang X, Ly KH, Greer HF, Reisner E (2020) Disparity of Cytochrome Utilization in Anodic and Cathodic Extracellular Electron Transfer Pathways of Geobacter sulfurreducens Biofilms. J Am Chem Soc 142:5194-5203.

34. Hogbom M, Huque Y, Sjoberg BM, Nordlund P (2002) Crystal structure of the diiron/radical protein of ribonucleotide reductase from Corynebacterium ammoniagenes. Biochemistry 41:1381-1389.

35. Holt SD, Piggott B, Ingledew WJ, Feiters MC, Diakun GP (1990) EXAFS of the type1 copper site of rusticyanin. FEBS Letters 269:117-121.

36. Inaba Y, West AC, Banta S (2020) Enhanced microbial corrosion of stainless steel by Acidithiobacillus ferrooxidans through the manipulation of substrate oxidation and overexpression of rus. Biotechnol Bioeng 117:3475-3485.

37. Ingledew WJ (1982) Thiobacillus Ferrooxidans the bioenergetics of an acidophilic chemolithotroph. Biochimica et Biophysica Acta (BBA) - Reviews on Bioenergetics 683:89-117.

38. Ingledew WJ, Cox JC, Halling PJ (1977) A proposed mechanism for energy conservation during Fe2+ oxidation by Thiobacillus ferro-oxidans: Chemiosmotic coupling to net $\mathrm{H}+$ influx. FEMS Microbiology Letters 2:193-197.

39. Jeans C, Singer SW, Chan CS, Verberkmoes NC, Shah M, Hettich RL, Banfield JF, Thelen MP (2008) Cytochrome 572 is a conspicuous membrane protein with iron oxidation activity purified directly from a natural acidophilic microbial community. ISME J 2:542-550.

40. Kamisetty H, Ovchinnikov S, Baker D (2013) Assessing the utility of coevolutionbased residue-residue contact predictions in a sequence- and structure-rich era. Proceedings of the National Academy of Sciences 110:15674-15679. 
41. Kappler A, Bryce C, Mansor M, Lueder U, Byrne JM, Swanner ED (2021) An evolving view on biogeochemical cycling of iron. Nat Rev Microbiol.

42. Kernan T, Majumdar S, Li X, Guan J, West AC, Banta S (2016) Engineering the iron-oxidizing chemolithoautotroph Acidithiobacillus ferrooxidans for biochemical production. Biotechnol Bioeng 113:189-197.

43. Kilambi KP, Gray JJ (2012) Rapid calculation of protein pKa values using Rosetta. Biophys J 103:587-595.

44. Koehler Leman J, Ulmschneider MB, Gray JJ (2015) Computational modeling of membrane proteins. Proteins 83:1-24.

45. Kotloski NJ, Gralnick JA (2013) Flavin electron shuttles dominate extracellular electron transfer by Shewanella oneidensis. mBio 4.

46. Kozakov D, Hall DR, Xia B, Porter KA, Padhorny D, Yueh C, Beglov D, Vajda S (2017) The ClusPro web server for protein-protein docking. Nat Protoc 12:255278.

47. Kucera J, Lochman J, Bouchal P, Pakostova E, Mikulasek K, Hedrich S, Janiczek O, Mandl M, Johnson DB (2020) A Model of Aerobic and Anaerobic Metabolism of Hydrogen in the Extremophile Acidithiobacillus ferrooxidans. Front Microbiol 11:610836.

48. Kucera J, Pakostova E, Lochman J, Janiczek O, Mandl M (2016) Are there multiple mechanisms of anaerobic sulfur oxidation with ferric iron in Acidithiobacillus ferrooxidans? Res Microbiol 167:357-366.

49. Kucera J, Pakostova E, Lochman J, Janiczek O, Mandl M (2016) Are there multiple mechanisms of anaerobic sulfur oxidation with ferric iron in Acidithiobacillus ferrooxidans? Res Microbiol 167:357-366.

50. Li TF, Painter RG, Ban B, Blake RC, 2nd (2015) The Multicenter Aerobic Iron Respiratory Chain of Acidithiobacillus ferrooxidans Functions as an Ensemble with a Single Macroscopic Rate Constant. J Biol Chem 290:18293-18303.

51. Li X, Mercado R, Berlinger S, Banta S, West AC (2014) Engineering Acidithiobacillus ferrooxidans growth media for enhanced electrochemical processing. AIChE Journal 60:4008-4013.

52. Liang N, Pielak GJ, Mauk AG, Smith M, Hoffman BM (1987) Yeast cytochrome c with phenylalanine or tyrosine at position 87 transfers electrons to (zinc cytochrome c peroxidase) + at a rate ten thousand times that of the serine-87 or glycine-87 variants. Proc Natl Acad Sci U S A 84:1249-1252.

53. Lin YF, Cheng CW, Shih CS, Hwang JK, Yu CS, Lu CH (2016) MIB: Metal IonBinding Site Prediction and Docking Server. J Chem Inf Model 56:2287-2291.

54. Liu W, Lin J, Pang X, Mi S, Cui S, Lin J (2013) Increases of ferrous iron oxidation activity and arsenic stressed cell growth by overexpression of Cyc2 in Acidithiobacillus ferrooxidans ATCC19859. Biotechnol Appl Biochem 60:623-628.

55. Liu Y, Fredrickson JK, Zachara JM, Shi L (2015) Direct involvement of ombB, omaB, and omcB genes in extracellular reduction of $\mathrm{Fe}(\mathrm{III})$ by Geobacter sulfurreducens PCA. Front Microbiol 6:1075.

56. Liu Y, Wang Z, Liu J, Levar C, Edwards MJ, Babauta JT, Kennedy DW, Shi Z, Beyenal H, Bond DR, Clarke TA, Butt JN, Richardson DJ, Rosso KM, Zachara JM, Fredrickson JK, Shi L (2014) A trans-outer membrane porin-cytochrome 
protein complex for extracellular electron transfer by Geobacter sulfurreducens PCA. Environ Microbiol Rep 6:776-785.

57. Lobley A, Sadowski MI, Jones DT (2009) pGenTHREADER and pDomTHREADER: new methods for improved protein fold recognition and superfamily discrimination. Bioinformatics 25:1761-1767.

58. Lomize MA, Pogozheva ID, Joo H, Mosberg HI, Lomize AL (2012) OPM database and PPM web server: resources for positioning of proteins in membranes. Nucleic Acids Res 40:D370-376.

59. Lyskov S, Chou FC, Conchuir SO, Der BS, Drew K, Kuroda D, Xu J, Weitzner BD, Renfrew PD, Sripakdeevong P, Borgo B, Havranek JJ, Kuhlman B, Kortemme T, Bonneau R, Gray JJ, Das R (2013) Serverification of molecular modeling applications: the Rosetta Online Server that Includes Everyone (ROSIE). PLoS One 8:e63906.

60. Madeira F, Park YM, Lee J, Buso N, Gur T, Madhusoodanan N, Basutkar P, Tivey ARN, Potter SC, Finn RD, Lopez R (2019) The EMBL-EBI search and sequence analysis tools APIs in 2019. Nucleic Acids Res 47:W636-W641.

61. Malvankar NS, Vargas M, Nevin KP, Franks AE, Leang C, Kim BC, Inoue K, Mester T, Covalla SF, Johnson JP, Rotello VM, Tuominen MT, Lovley DR (2011) Tunable metallic-like conductivity in microbial nanowire networks. Nat Nanotechnol 6:573-579.

62. McAllister SM, Polson SW, Butterfield DA, Glazer BT, Sylvan JB, Chan CS (2020) Validating the Cyc2 Neutrophilic Iron Oxidation Pathway Using Meta-omics of Zetaproteobacteria Iron Mats at Marine Hydrothermal Vents. mSystems 5.

63. Methe BA, Nelson KE, Eisen JA, Paulsen IT, Nelson W, Heidelberg JF, Wu D, Wu M, Ward N, Beanan MJ, Dodson RJ, Madupu R, Brinkac LM, Daugherty SC, DeBoy RT, Durkin AS, Gwinn M, Kolonay JF, Sullivan SA, Haft DH, Selengut J, Davidsen TM, Zafar N, White O, Tran B, Romero C, Forberger HA, Weidman J, Khouri H, Feldblyum TV, Utterback TR, Van Aken SE, Lovley DR, Fraser CM (2003) Genome of Geobacter sulfurreducens: metal reduction in subsurface environments. Science 302:1967-1969.

64. Meuzelaar H, Vreede J, Woutersen S (2016) Influence of Glu/Arg, Asp/Arg, and Glu/Lys Salt Bridges on alpha-Helical Stability and Folding Kinetics. Biophys J 110:2328-2341.

65. Modi N, Ganguly S, Bárcena-Uribarri I, Benz R, van den Berg B, Kleinekathöfer U (2015) Structure, Dynamics, and Substrate Specificity of the OprO Porin from Pseudomonas aeruginosa. Biophysical Journal 109:1429-1438.

66. Mukhopadhyay BP, Ghosh B, Bairagya HR, Nandi TK, Chakrabarti B, Bera AK (2008) Molecular modeling of the ternary complex of Rusticyanin-cytochrome c4cytochrome oxidase: an insight to possible $\mathrm{H}$-bond mediated recognition and electron transfer reaction in T. ferrooxidans. J Biomol Struct Dyn 25:543-551.

67. Nemati M, Harrison STL, Hansford GS, Webb C (1998) Biological oxidation of ferrous sulphate by Thiobacillus ferrooxidans: a review on the kinetic aspects. Biochemical Engineering Journal 1:171-190.

68. Nishida Y, Kino K, Kida S (1987) Crystal structures of low- and high-spin iron(III) complexes with quadridentate Schiff bases. Journal of the Chemical Society, Dalton Transactions:1157. 
69. O'Donnell JP, Phillips BP, Yagita Y, Juszkiewicz S, Wagner A, Malinverni D, Keenan RJ, Miller EA, Hegde RS (2020) The architecture of EMC reveals a path for membrane protein insertion. Elife 9.

70. Okamoto A, Hashimoto K, Nealson KH, Nakamura R (2013) Rate enhancement of bacterial extracellular electron transport involves bound flavin semiquinones. Proc Natl Acad Sci U S A 110:7856-7861.

71. Ovchinnikov S, Kamisetty H, Baker D (2014) Robust and accurate prediction of residue-residue interactions across protein interfaces using evolutionary information. Elife 3:e02030.

72. Priya A, Hait S (2018) Extraction of metals from high grade waste printed circuit board by conventional and hybrid bioleaching using Acidithiobacillus ferrooxidans. Hydrometallurgy 177:132-139.

73. Pronk JT, Bruyn JCd, Bos P, Kuenen JG (1992) Anaerobic growth of Thiobacillus ferrooxidans. Appl Environ Microbiol 58:2227-2230.

74. Quatrini R, Appia-Ayme C, Denis Y, Jedlicki E, Holmes DS, Bonnefoy V (2009) Extending the models for iron and sulfur oxidation in the extreme acidophile Acidithiobacillus ferrooxidans. BMC Genomics 10:394.

75. Randall A, Cheng J, Sweredoski M, Baldi P (2008) TMBpro: secondary structure, beta-contact and tertiary structure prediction of transmembrane beta-barrel proteins. Bioinformatics 24:513-520.

76. Reguera G, McCarthy KD, Mehta T, Nicoll JS, Tuominen MT, Lovley DR (2005) Extracellular electron transfer via microbial nanowires. Nature 435:1098-1101.

77. Rohwerder T, Gehrke T, Kinzler K, Sand W (2003) Bioleaching review part A: progress in bioleaching: fundamentals and mechanisms of bacterial metal sulfide oxidation. Appl Microbiol Biotechnol 63:239-248.

78. Ronk M, Shively JE, Shute EA, Blake RC, 2nd (1991) Amino acid sequence of the blue copper protein rusticyanin from Thiobacillus ferrooxidans. Biochemistry 30:9435-9442.

79. Slusky JS (2017) Outer membrane protein design. Curr Opin Struct Biol 45:45-52.

80. Tazhigulov RN, Gayvert JR, Wei M, Bravaya KB (2019) eMap: A Web Application for Identifying and Visualizing Electron or Hole Hopping Pathways in Proteins. J Phys Chem B 123:6946-6951.

81. Tian W, Lin M, Tang K, Liang J, Naveed H (2018) High-resolution structure prediction of beta-barrel membrane proteins. Proc Natl Acad Sci U S A 115:1511-1516.

82. Uziela K, Menendez Hurtado D, Shu N, Wallner B, Elofsson A (2017) ProQ3D: improved model quality assessments using deep learning. Bioinformatics 33:1578-1580.

83. Uziela K, Shu N, Wallner B, Elofsson A (2016) ProQ3: Improved model quality assessments using Rosetta energy terms. Sci Rep 6:33509.

84. Vajda S, Yueh C, Beglov D, Bohnuud T, Mottarella SE, Xia B, Hall DR, Kozakov D (2017) New additions to the ClusPro server motivated by CAPRI. Proteins 85:435-444.

85. Valdes J, Pedroso I, Quatrini R, Dodson RJ, Tettelin H, Blake R, 2nd, Eisen JA, Holmes DS (2008) Acidithiobacillus ferrooxidans metabolism: from genome sequence to industrial applications. BMC Genomics 9:597. 
86. Vorobieva AA, White P, Liang B, Horne JE, Bera AK, Chow CM, Gerben S, Marx S, Kang A, Stiving AQ, Harvey SR, Marx DC, Khan GN, Fleming KG, Wysocki VH, Brockwell DJ, Tamm LK, Radford SE, Baker D (2021) De novo design of transmembrane beta barrels. Science 371 .

87. Walter RL, Ealick SE, Friedman AM, Blake RC, 2nd, Proctor P, Shoham M (1996) Multiple wavelength anomalous diffraction (MAD) crystal structure of rusticyanin: a highly oxidizing cupredoxin with extreme acid stability. J Mol Biol 263:730-751.

88. Wang C, Vernon R, Lange O, Tyka M, Baker D (2010) Prediction of structures of zinc-binding proteins through explicit modeling of metal coordination geometry. Protein Sci 19:494-506.

89. Weinstein JY, Elazar A, Fleishman SJ (2019) A lipophilicity-based energy function for membrane-protein modelling and design. PLoS Comput Biol 15:e1007318.

90. Wheaton G, Counts J, Mukherjee A, Kruh J, Kelly R (2015) The Confluence of Heavy Metal Biooxidation and Heavy Metal Resistance: Implications for Bioleaching by Extreme Thermoacidophiles. Minerals 5:397-451.

91. White GF, Edwards MJ, Gomez-Perez L, Richardson DJ, Butt JN, Clarke TA (2016) Mechanisms of Bacterial Extracellular Electron Exchange. Adv Microb Physiol 68:87-138.

92. Williams CJ, Headd JJ, Moriarty NW, Prisant MG, Videau LL, Deis LN, Verma V, Keedy DA, Hintze BJ, Chen VB, Jain S, Lewis SM, Arendall WB, 3rd, Snoeyink J, Adams PD, Lovell SC, Richardson JS, Richardson DC (2018) MolProbity: More and better reference data for improved all-atom structure validation. Protein Sci 27:293-315.

93. Xiao X, Yu HQ (2020) Molecular mechanisms of microbial transmembrane electron transfer of electrochemically active bacteria. Curr Opin Chem Biol 59:104-110.

94. Xu S, Jangir Y, El-Naggar MY (2016) Disentangling the roles of free and cytochrome-bound flavins in extracellular electron transport from Shewanella oneidensis MR-1. Electrochimica Acta 198:49-55.

95. Yamauchi O, Odani A, Takani M (2002) Metal-amino acid chemistry. Weak interactions and related functions of side chain groups. J Chem Soc, Dalton Trans:3411-3421.

96. Yarov-Yarovoy V, Schonbrun J, Baker D (2006) Multipass membrane protein structure prediction using Rosetta. Proteins 62:1010-1025.

97. Yarzabal A, Brasseur G, Ratouchniak J, Lund K, Lemesle-Meunier D, DeMoss JA, Bonnefoy V (2002) The high-molecular-weight cytochrome c Cyc2 of Acidithiobacillus ferrooxidans is an outer membrane protein. J Bacteriol 184:313317.

98. Zachariae U, Kluhspies T, De S, Engelhardt H, Zeth K (2006) High resolution crystal structures and molecular dynamics studies reveal substrate binding in the porin Omp32. J Biol Chem 281:7413-7420.

99. Zhan Y, Yang M, Zhang S, Zhao D, Duan J, Wang W, Yan L (2019) Iron and sulfur oxidation pathways of Acidithiobacillus ferrooxidans. World J Microbiol Biotechnol 35:60.

100. Zheng H, Chordia MD, Cooper DR, Chruszcz M, Muller P, Sheldrick GM, Minor W (2014) Validation of metal-binding sites in macromolecular structures with the CheckMyMetal web server. Nat Protoc 9:156-170. 
bioRxiv preprint doi: https://doi.org/10.1101/2021.03.22.436458; this version posted March 22, 2021. The copyright holder for this preprint (which was not certified by peer review) is the author/funder, who has granted bioRxiv a license to display the preprint in perpetuity. It is made available under aCC-BY-NC 4.0 International license.

101. Zheng H, Chruszcz M, Lasota P, Lebioda L, Minor W (2008) Data mining of metal ion environments present in protein structures. J Inorg Biochem 102:1765-1776. 


\section{Figures}

A

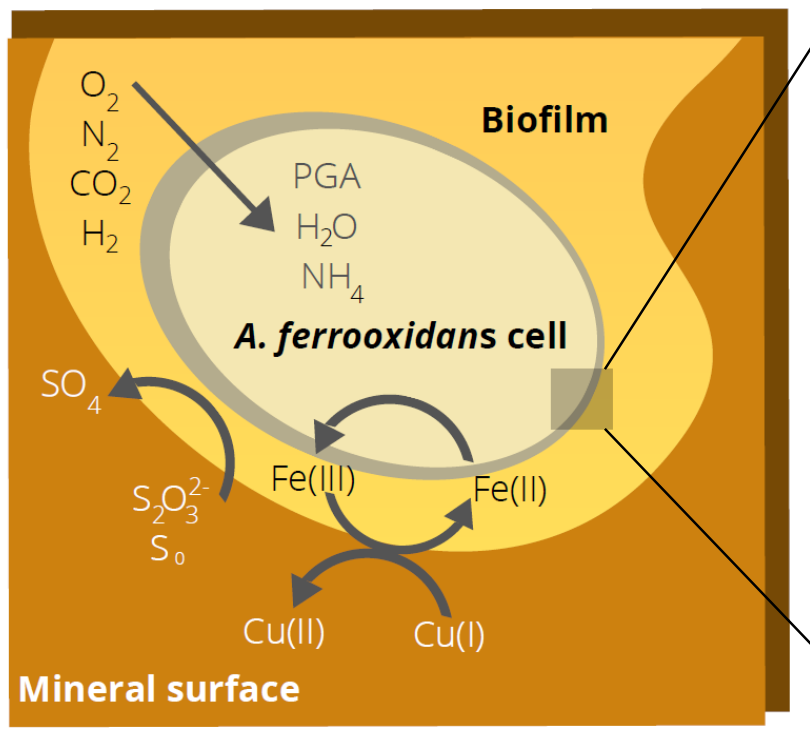

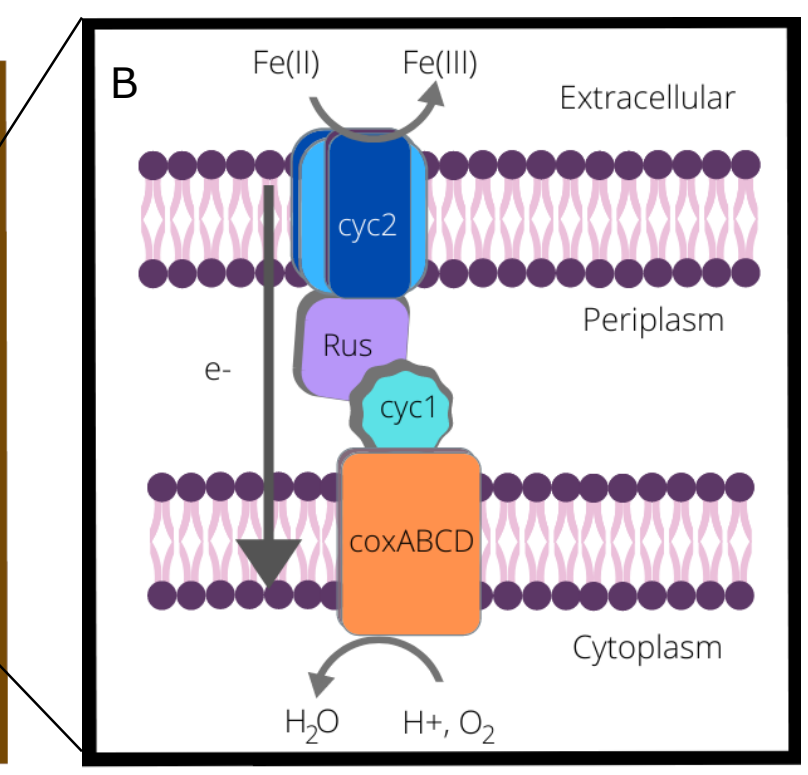

Figure 1. Overview of metal oxidation by A. ferrooxidans. A. Overview of $A$. ferrooxidans metabolism when growing on ores containing sulfide minerals such as chalcopyrite ( $\left.\mathrm{CuFeS}_{2}\right)$. The bacterium oxidizes iron and reduced sulfur compounds, resulting in the solubilization of copper or other commercially valuable metal targets. Carbon dioxide is fixed via the Calvin cycle to 3-phosphoglycerate (PGA). B. Diagram of the electron transfer pathway used for extracellular iron oxidation. Electrons are transferred from the extracellular matrix, through the outer membrane (Cyc2), the periplasmic space (Rus and Cyc1) to coxABCD in the inner membrane. This is a simplified model of the downhill electron transfer pathway, as other periplasmic proteins may also be involved in this process. Under some conditions, electron transfer can be reversed, and iron reduction can also occur. 


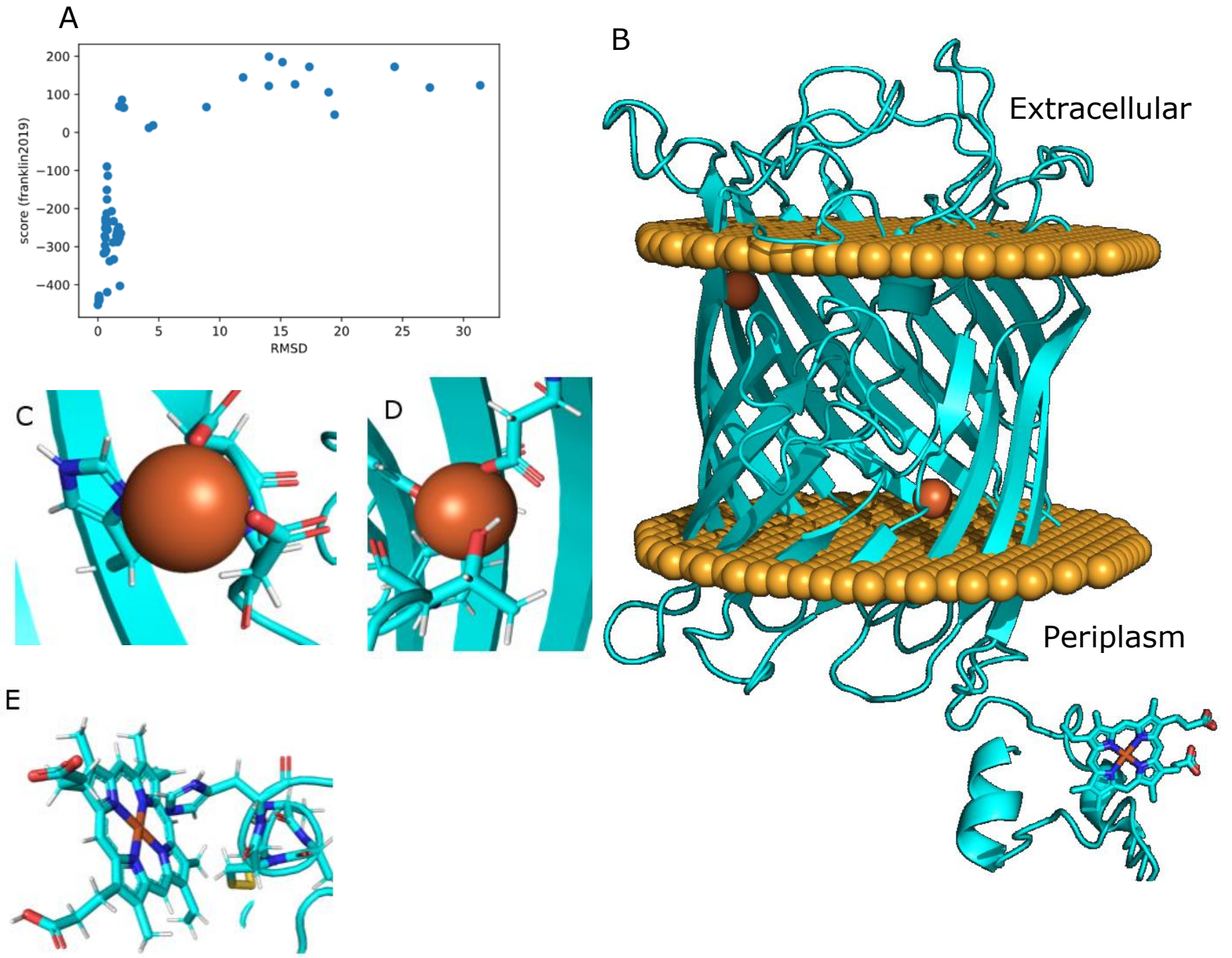

Figure 2. Characterization of modeled Cyc2 protein and interactions with ligands: A: Funnel plot for one hundred Cyc2 modeling trajectories using the modified set of membrane weights. The c-alpha RMSD was measured in reference to the lowestscoring structure. B: Lowest-scoring Cyc2 protein model. Disks of spheres represent the phospholipid heads on the outer membrane where the top of the protein protrudes into the extracellular matrix and bottom of the protein (including the heme group) is in the periplasm. C: Square planar iron chelating geometry for residues H119, D137, D138. D: Tetrahedral iron binding geometry for Y262 and D308. E. Binding interaction between $\mathrm{H} 16$ and heme $\mathrm{c}$. 
A

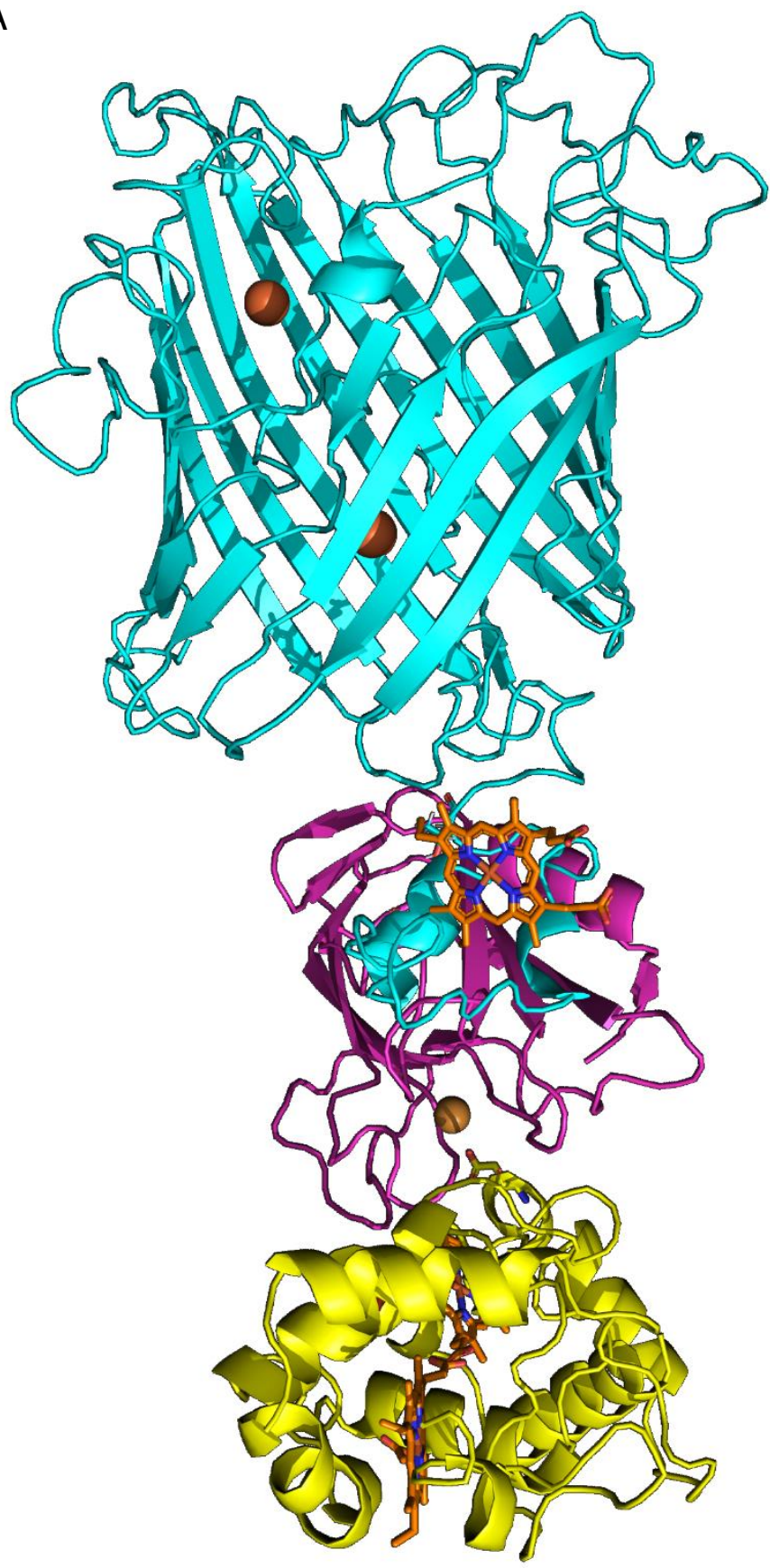

B

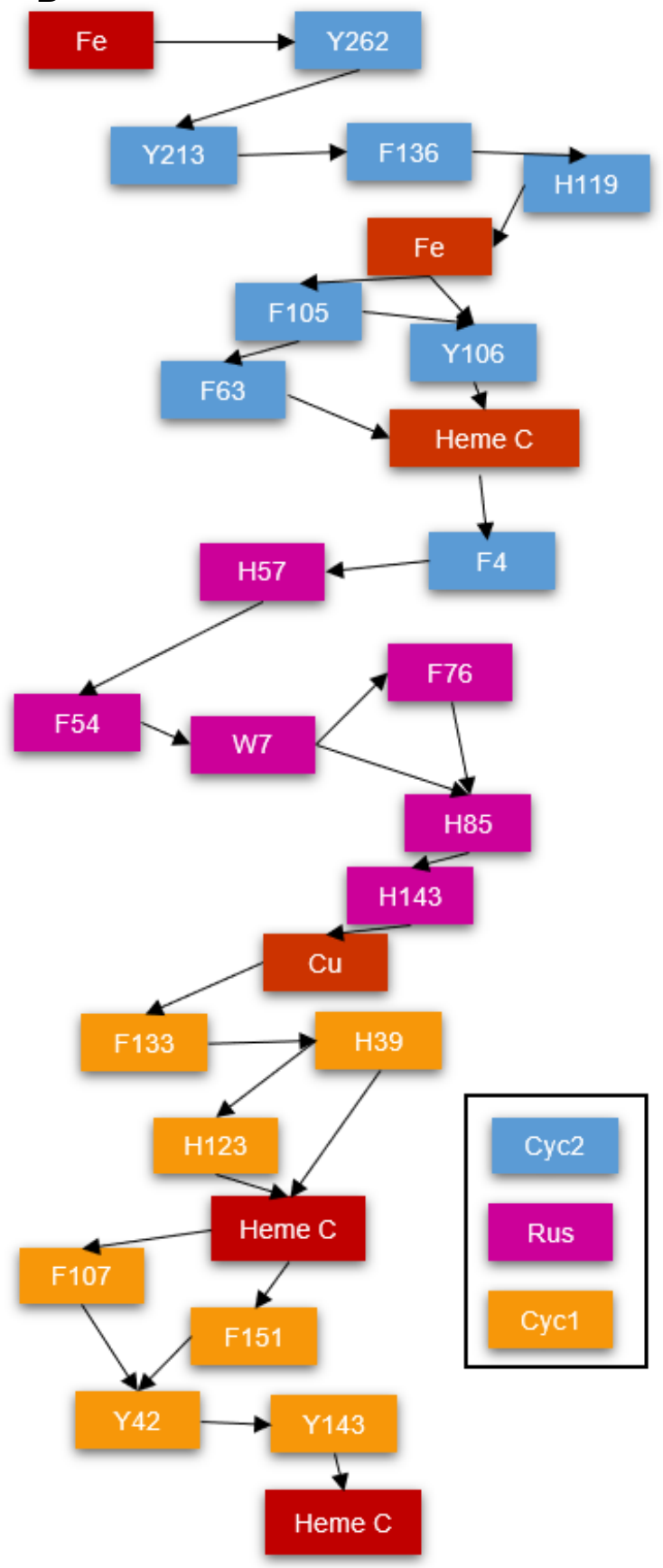

Figure 3. Predicted protein-protein interactions supporting the electron transport chain: A: Docked models of the Cyc2-rusticyanin-Cyc1 pathway (where carbon backbone of Cyc2 is blue, rusticyanin in magenta, and Cyc1 is in yellow). Hemes, iron ions, and copper ions are indicated in sepia. B: Predicted electron hopping pathway for electron transfer from the exterior of the cell into the periplasm of $A$. ferrooxidans. 

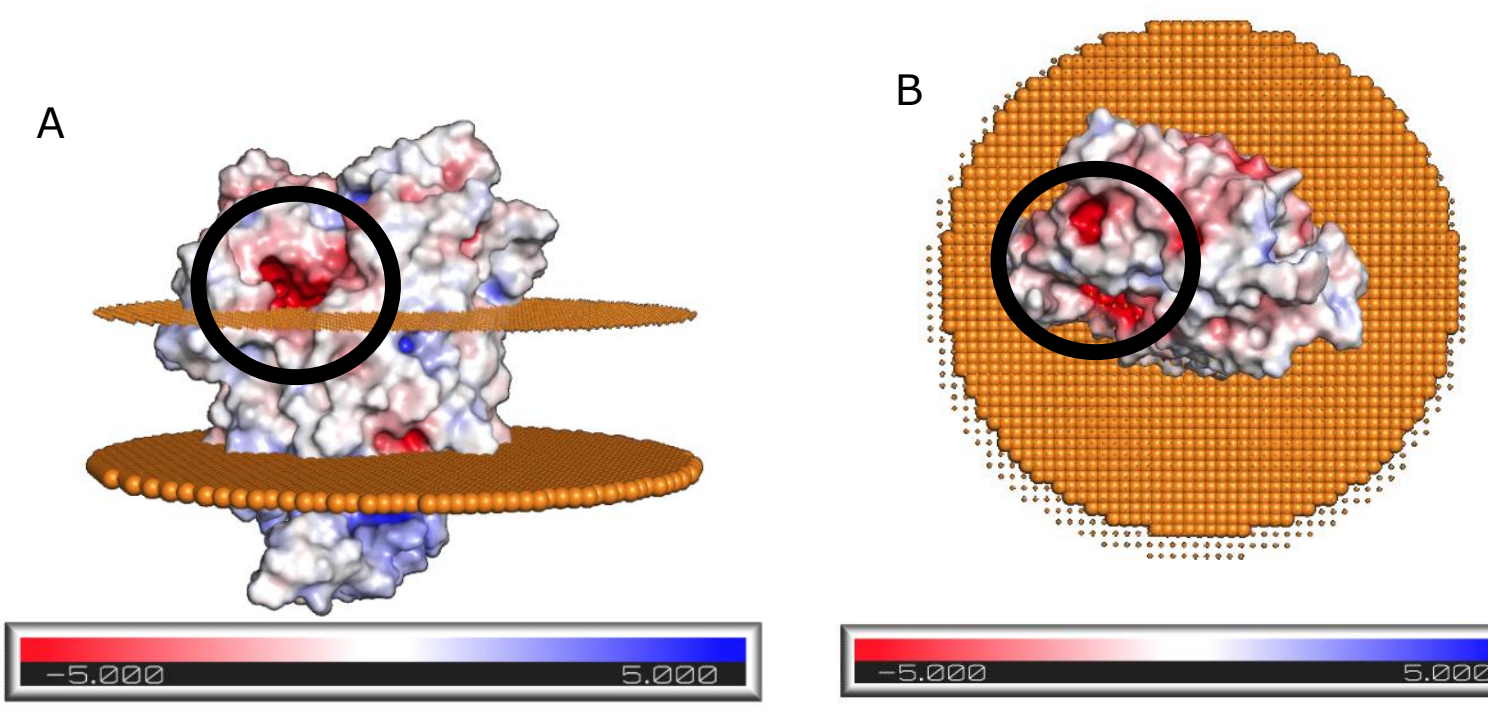

C

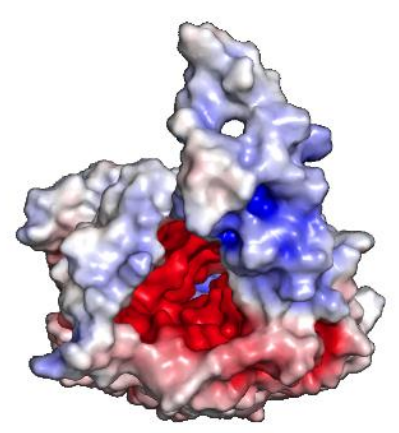

$-5.000$

Figure 4. Poisson-Boltzmann potential identifies possible iron binding pocket on the extracellular region of Cyc2: Poisson-Boltzmann potential for Cyc2, where blue indicates regions of positive potential $(>+5 \mathrm{kT} / \mathrm{e})$ whereas red depicts negative potential $(<-5 \mathrm{kT} / \mathrm{e})$. A: labeled potential viewed from the side, where the upper disk represents phospholipid heads on the outer membrane facing the extracellular matrix and bottom disk represents the phospholipids facing the periplasm. There exists a region of high negative potential (circled in black) that may serve as a possible iron binding site. B: Labeled potential as viewed from above, showing the protein structure protruding from the cell. Regions of high negative potential are circled in black. C: Labeled potential as viewed from below the membrane, as would be observed from inside the cell. An annular space exists within the protein, inside which is predicted to have a high negative potential 